\title{
Prevenzione dell'epatite B nei centri dialisi: la vaccinazione antiepatite $B$
}

\author{
M. Del Prete
}

Servizio Nefrologia e Dialisi, Ospedale S. Paolo - Milano

] 2

epatite B ha rappresentato, in un passato ancora recente, una importante complicanza per i pazienti in trattamento dialitico, in relazione alle frequenti trasfusioni, alla necessità di sottoporsi ad indagini $\mathrm{e}$ manovre invasive ed alla possibilità di essere contagiati da altri pazienti portatori del virus. Inoltre, il paziente uremico presenta un deficit dell'immunità cellulare ed umorale che lo rende più suscettibile alle infezioni e condiziona, nel caso specifico dell'epatite B, un'espressione spesso subclinica della malattia con persistenza del virus in circa il $60 \%$ dei soggetti infetti che divengono a loro volta fonte di contagio (1).

Nel 1979 l'EDTA (2) denunciava la contaminazione da parte del virus del $50 \%$ dei centri dialisi Europei, con il $22 \%$ dei pazienti portatori del virus e 750 casi di epatite $B$ tra il personale sanitario (3). Nel 1985 rispetto al 1980, sempre riferendosi ai dati dell'EDTA, si può notare un'iniziale inversione di tendenza con una diminuzione dell'incidenza per anno dei casi di epatite $B$ tra $i$ pazienti ed un aumento relativo delle epatiti NANB (Fig. 1). Oggi, nonostante la mancanza di misure terapeutiche efficaci, il problema delle epatiti $\mathbf{B}$ nei centri dialisi è stato notevolmente ridimensionato. Un'accurata selezione dei donatori, la recente disponibilità di eritropoietina umana ricombinante che ha ridotto l'uso delle trasfusioni, l'individuazione e ove possibile l'isolamento dei pazienti infetti, l'immunizzazione passiva con globuline sieriche dei soggetti accidentalmente esposti ad una fonte di contagio, hanno contribuito a ridurre drasticamente l'incidenza delle epatiti $\mathbf{B}$. Ma non vi è dubbio che la misura preventiva più efficace sia rappresentata dalla vaccinazione.

\section{La vaccinazione $B$}

Negli ultimi dieci anni sono stati sperimentati in USA e in Europa vaccini plasma derivati, ottenuti cioè dal plasma di soggetti infetti $\mathrm{e}$ contenenti antigeni virali sottoposti a processi di purificazione ed inattivazione.

Attualmente sono disponibili in commercio un vaccino americano che viene somministrato per via intramuscolare alla dose di $40 \mathrm{mcg}$, al tempo 0 , dopo 1 e 6 mesi ed un vaccino francese, somministrato alla dose di $5 \mathrm{mcg}$ al tempo 0 , dopo 1 , 2 e 14 mesi.

Nel $95 \%$ dei soggetti sani si ottiene una soddisfacente produzione di anti-HBs che è l'anticorpo rivolto verso l'antigene di superficie del virus, con una funzione protettiva nei confronti dell'infezione (4). Viene considerato protettivo un titolo anticorpale superiore alle 10 $\mathrm{mUI} / \mathrm{ml}$ e la durata della protezione è di circa 5 anni con ampie variazioni individuali. È pertanto necessario un periodico controllo del titolo anticorpale per decidere quando effettuare la dose di richiamo. Gli effetti collaterali sono rari e di modesta entità: febbre, cefalea, 


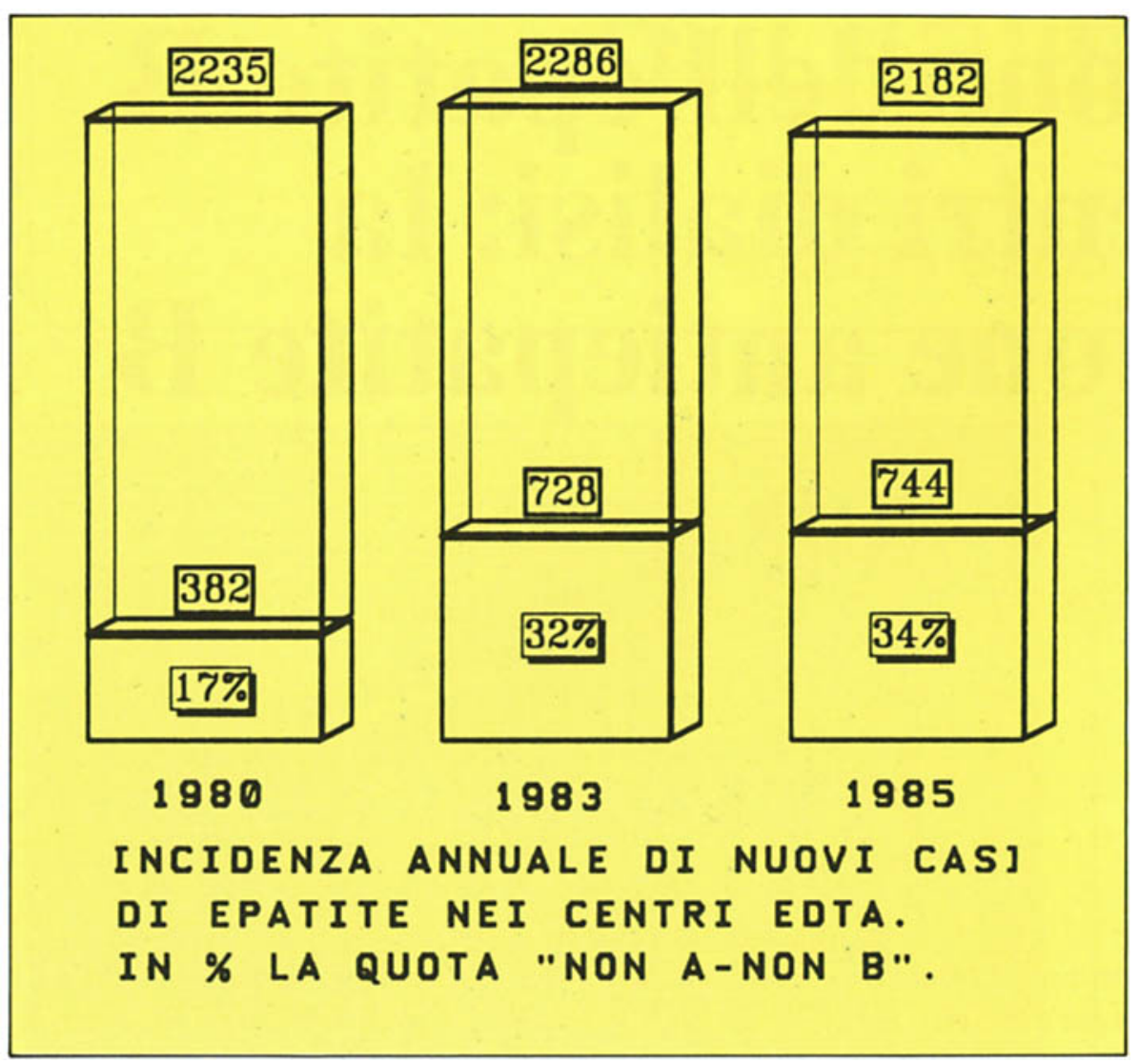

Fig. 1

nausea, dolore nella sede dell'iniezione.

Recentemente si è reso disponibile un vaccino ottenuto con la tecnica del DNA ricombinante. Il gene responsabile della produzione dell'HBsAg, che è l'antigene virale di superficie, viene inserito per mezzo di un plasmide, all'interno del DNA di un lievito. Le cellule del lievito divengono così in grado di produrre elevate quantità di HBsAg. Rispetto ai vaccini plasma derivati, i vaccini ricombinanti annullano il rischio di trasmissione di altri virus patogeni e consentono produzioni di vaccino praticamente illimitate e con una risposta anticorpale con una netta riduzione dei costi. Il vaccino ricombinante comporta una risposta anticorpale con un titolo lievemente inferiore rispetto ai plasma derivati ma altret- tanto efficace in termini protettivi $(5,6)$. Nei soggetti sani viene somministrato alla dose di $20 \mathrm{mcg}$ per via intramuscolare, al tempo 0 , dopo 1 e 6 mesi.

\section{La vaccinazione dei pazienti in dialisi}

I pazienti in dialisi presentano una
TAB. I
I pazienti in dialisi presentano una risposta al vaccino meno soddisfacente dei soggetti sani. Solo dal 50 al $70 \%$ dei soggetti vaccinati sviluppano un titolo anticorpale protettivo e con un'ampia variabilità secondo le casistiche esaminate (Tab. I).

Tale discordanza di dati è in parte attribuibile alla diversità dei vaccini e delle schedule vaccinali utilizzate dai vari Autori ed alla disomogeneità dei gruppi di pazienti studiati. Sono infatti state individuate una serie di variabili quali l'età, il sesso, il peso corporeo, la sede dell'iniezione che sembrano condizionare in modo più o meno decisivo la risposta anticorpale. In particolare, è stata osservata da numerosi Autori l'influenza dell'età sulla risposta anticorpale che è massima sotto i 30 anni e diminuisce nettamente dopo i 60 (7). Le donne hanno frequentemente titoli anticorpali più alti degli uomini (8) e i soggetti obesi presentano un deficit immunitario rispetto ai soggetti più magri, indipendentemente dall'uremia (9).

Inoltre, la somministrazione intramuscolare nel deltoide comporta una risposta migliore rispetto a quella del gluteo (10) ed è ancora dibattuta la scelta tra via intramuscolare ed intradermica (11).

Esaminiamo ora i passi da seguire per un corretto programma di vaccinazione.

\begin{tabular}{lcccccc}
\hline $\begin{array}{l}\text { Primo } \\
\text { Autore }\end{array}$ & Anno & $\begin{array}{c}\text { Pazienti } \\
\text { n. }\end{array}$ & Vaccino & $\begin{array}{c}\text { Dose } \\
(\mathrm{mcg})\end{array}$ & Schema & $\begin{array}{c}\text { Risposta } \\
\%\end{array}$ \\
\hline Stevens & 1984 & 660 & $\mathbf{P}$ & 40 & $0-1-6$ & 63 \\
Bommer & 1985 & 25 & $\mathbf{P}$ & 40 & $0-1-6$ & 54 \\
Kohler & 1985 & 99 & $\mathbf{P}$ & 40 & $0-1-6$ & 56.5 \\
Crosnier & 1985 & 43 & $\mathbf{P}$ & 40 & $0-1-6$ & 60 \\
Bruguera & 1987 & 36 & $\mathbf{R}$ & 40 & $0-1-2$ & 54 \\
& & 44 & $\mathbf{R}$ & 40 & $0-1-6$ & 56 \\
Tay & 1989 & 32 & $\mathbf{R}$ & 40 & $0-1-2-6-12$ & 83 \\
\hline
\end{tabular}




\section{RICHIESTE MARKERS EPATITE B}

\section{PAZIENTE INFETTO}

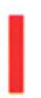

FASE ACUTA

\begin{tabular}{|l|c|c|c|c|c|c|}
\hline & HBsAg & HBsAg & \multicolumn{2}{|c|}{$\begin{array}{c}\text { Anti-HBe } \\
\text { lgM }\end{array}$} & $\begin{array}{c}\text { Anti- } \\
\text { HBG }\end{array}$ & $\begin{array}{c}\text { Anti- } \\
\text { HBs }\end{array}$ \\
\hline Precoce & + & - & - & - & - & - \\
\hline $\begin{array}{l}\text { Viremia } \\
(>14 \mathrm{gg})\end{array}$ & + & + & + & - & - & - \\
\hline Tardiva & + & - & + & + & + & - \\
\hline
\end{tabular}

LA "FINESTRA" DELL'ANTI-CORE

\begin{tabular}{|c|c|c|c|c|c|}
\hline HBsAg & HBsAg & \multicolumn{2}{|c|}{$\begin{array}{c}\text { Anti-HBe } \\
\text { lgM }\end{array}$} & $\begin{array}{c}\text { Anti- } \\
\text { HBe }\end{array}$ & $\begin{array}{c}\text { Anti- } \\
\text { HBs }\end{array}$ \\
\hline- & - & + & + & + & - \\
\hline
\end{tabular}

\section{CONVALESCENZA}

\begin{tabular}{|c|c|c|c|c|c|}
\hline HBsAg & HBsAg & \multicolumn{2}{|c|}{$\begin{array}{c}\text { Anti-HBe } \\
\text { lgM }\end{array}$} & $\begin{array}{c}\text { Anti- } \\
\text { HBe }\end{array}$ & $\begin{array}{c}\text { Anti- } \\
\text { HBs }\end{array}$ \\
\hline- & - & - & + & + & + \\
\hline
\end{tabular}

PAZIENTE IMMUNE PER PRECEDENTE VACCINAZIONE

IMMUNIZZAZIONE

\begin{tabular}{|c|c|c|c|c|c|}
\hline HBsAg & HBsAg & \multicolumn{2}{|c|}{$\begin{array}{c}\text { Anti-HBe } \\
\text { lgM }\end{array}$} & $\begin{array}{c}\text { Anti- } \\
\text { HBe }\end{array}$ & $\begin{array}{c}\text { Anti- } \\
\text { HBs }\end{array}$ \\
\hline- & - & - & - & - & + \\
\hline
\end{tabular}

PAZIENTE SUSCETTIBILE DA VACCINARE

\begin{tabular}{|c|c|c|c|c|c|}
\hline HBsAg & HBsAg & \multicolumn{2}{|c|}{$\begin{array}{c}\text { Anti-HBe } \\
\text { IgM }\end{array}$} & $\begin{array}{c}\text { Anti- } \\
\text { HBe }\end{array}$ & $\begin{array}{c}\text { Anti- } \\
\text { HBs }\end{array}$ \\
\hline- & - & - & - & - & - \\
\hline
\end{tabular}

\section{QUADRO RIASSUNTIVO DELLA SEQUENZA DEI MARKERS}

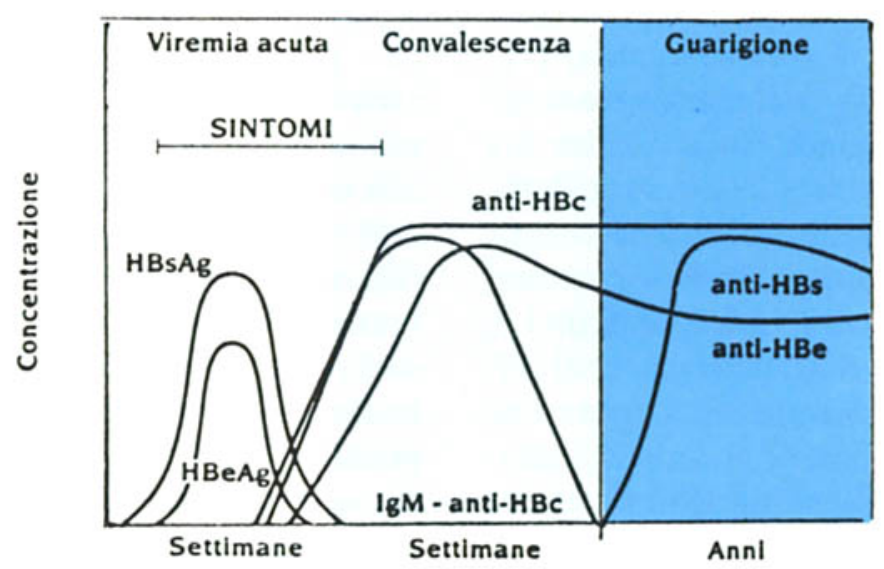

Fig. 2

\section{Selezione dei pazienti}

Il primo pasșo consiste nella corretta selezione dei pazienti che dovranno essere vaccinati.

L'antiHBc è l'anticorpo rivolto verso il "core", verso la parte centrale del virus; compare in corso di infezione, non ha valore protettivo e permane per molti anni dopo l'eliminazione del virus dall'organismo, probabilmente per tutta la vita. L'anti-HBs è, come si è detto, l'anticorpo rivolto verso l'antigene di superficie del virus, ha significato protettivo e la sua comparsa corrisponde all'eliminazione del virus dall'organismo, permane per molti anni ma tende, nella maggior parte dei casi, a diminuire prima dell'anti-HBc (Fig. 2). La presenza in un soggetto HBsAg negativo di entrambi gli anticorpi è indicativa di sieroconversione, ossia di un pregresso contatto con il virus che è stato successivamente eliminato da un'adeguata risposta anticorpale. Ovviamente questi soggetti non vanno vaccinati. La presenza in un soggetto HBsAg negativo di uno solo degli anticorpi può avere significati diversi. Il solo anti-HBc in assenza di anti-HBs, può rappresentare un falso positivo, ma più frequentemente questi pazienti risponderanno alla vaccinazione con una rapida produzione di anti-HBs ad alto titolo, ossia con una risposta che viene detta anamnestica ed è indicativa di una immunità già presente. L'anti-HBs isolato ha invece un significato più controverso, una parte dei soggetti con questo pattern anticorpale, circa il $45 \%$ (12) possono presentare se vaccinati $u$ na risposta anamnestica ma nei casi restanti la risposta rimane di dubbia interpretazione. È probabilmente opportuno, almeno nei gruppi ad altro rischio, una valutazione prevaccinale di entrambi gli anticorpi e la vaccinazione dei casi dubbi considerando l'assoluta innocuità della somministrazione di 
vaccino a soggetti già immuni (12).

\section{Lo schema di vaccinazione}

Per cercare di aumentare la risposta anticorpale dei pazienti in dialisi, sono state studiate diverse strategie. Si è associato il vaccino allo zinco (13), all'interferone gamma (14), ad immunostimolanti quali la timopentina (15) allo scopo di migliorarne la immunogenicità, con risultati non sempre soddisfacenti. Più efficace è stata la sperimentazione di schemi vaccinali modificati nel senso di un maggior quantitativo di vaccino e/o di un maggior numero di somministrazioni (16, 17) (Tab. II). Attualmente lo schema raccomandato dal Center for Disease Control è la somministrazione di $40 \mathrm{mcg}$ (una dose doppia rispetto ai soggetti sani) di vaccino ricombinante al tempo 0 e dopo 1,6 mesi per via intramuscolare nel deltoide, utilizzando un vaccino che contenga come adiuvante un quantitativo di alluminio non superiore a $1.25 \mathrm{mg}$ per dose, in relazione ai problemi connessi con la tossicità da alluminio (18). Secondo alcuni

TAB. II SCHEMI DI VACCINAZIONE

\begin{tabular}{|c|c|c|c|c|c|c|}
\hline \multirow[b]{2}{*}{ Dose (mcg) } & \multirow{2}{*}{$\begin{array}{c}\begin{array}{c}\text { Vaccino } \\
\text { Francese }\end{array} \\
5\end{array}$} & \multirow{2}{*}{$\begin{array}{c}\begin{array}{c}\text { Vaccino } \\
\text { Americano }\end{array} \\
20\end{array}$} & \multicolumn{2}{|c|}{$\operatorname{Recombivax}^{\mathbf{0}}$} & \multicolumn{2}{|c|}{ Engerix $B$} \\
\hline & & & 20 & $40\left(^{*}\right)$ & 20 & $40\left(^{*}\right)$ \\
\hline $\begin{array}{l}\text { mesi } \\
0\end{array}$ & $\mathbf{X}$ & $\mathbf{X}$ & & $\mathbf{X}$ & & $\mathbf{X}$ \\
\hline 1 & $\mathbf{X}$ & $\mathbf{X}$ & & $\mathbf{X}$ & & $\mathbf{X}$ \\
\hline 2 & $\mathrm{X}$ & $X\left({ }^{*}\right)$ & & $X\left({ }^{*}\right)$ & & $X(*)$ \\
\hline 6 & & $\mathbf{X}$ & & $\mathbf{X}$ & & $\mathbf{X}$ \\
\hline \multicolumn{7}{|l|}{12} \\
\hline 14 & $\mathrm{X}$ & & & & & \\
\hline
\end{tabular}

$\operatorname{mcg}\left({ }^{*}\right)$ : dosaggio modificato per pazienti in dialisi

$\mathrm{X}\left({ }^{*}\right)$ : somministrazione aggiuntiva per pazienti in dialisi

- 40 mcg di Recombivax $=4$ dosi, Alluminio $>2 \mathrm{mg}$

(limite consigliato $1.25 \mathrm{mg}$ )
Autori (19) la somministrazione di una dose anche al $2^{\circ}$ mese comporterebbe una sieroconversione in circa $1^{\prime} 80 \%$ dei pazienti in dialisi.

Nei soggetti in dialisi la titolazione dell'antiHBs dovrà essere effettuata almeno ogni 6 mesi dopo il ciclo vaccinale di base, somministrando una dose vaccinale di rinforzo quando il titolo risulterà inferiore alle $10 \mathrm{mUI} / \mathrm{ml}$.

\section{Vaccinazione e trapianto: un possi- bile conflitto}

I vaccini che contengono stabilizzatori, preservanti o sostanze derivate dai sistemi di coltura per la crescita dei virus, potrebbero scatenare risposte immunologiche aspecifiche. Pertanto una vaccinazione effettuata dopo o immediatamente prima di un trapianto potrebbe favorire reazioni di rigetto. Tale rischio è presumibilmente già controllato dalla terapia immunosoppressiva. È comunque buona norma terminare il ciclo vaccinale prima che il paziente venga inserito in lista attiva di trapianto in modo tale da annullare ogni possibile ri-

schio di attivazione immunologica. Inoltre la protezione nei confronti del virus $\mathrm{B}$, permetterà di utilizzare reni provenienti da donatori HBsAg positivi e di ridurre il rischio di sviluppare una epatite B dopo il trapianto (20).

\section{Strategie per il futuro}

1) Il paziente uremico presenta un deficit della risposta immunitaria molto prima della sua entrata in dialisi. Tuttavia la somministrazione di vaccino a pazienti uremici nelle fasi precoci della malattia, ha permesso di ottenere risposte anticorpali più soddisfacenti rispetto ai soggetti già in trattamento dialitico $(21,22)$. Tale profilassi precoce permetterà l'immissione in dialisi di pazienti già protetti che potranno essere trattati anche insieme a pazienti $\mathrm{HBsAg}$ positivi.

2) L'eritropoietina, che è entrata a far parte del bagaglio terapeutico del nefrologo, sembra migliorare la risposta immunitaria del paziente uremico. Uno studio recente (23) ha dimostrato che i pazienti in trattamento con eritropoietina presentano una risposta anticorpale alla vaccinazione migliore rispetto ai pazienti non trattati.

3) Il vaccino antiepatite $B$ è disponibile da diversi anni. Tuttavia la sua diffusione, soprattutto tra il personale sanitario, incontra ancora inspiegabili resistenze. La disponibilità di un vaccino ricombinante dovrebbe aver eliminato le perplessità, probabilmente infondate, legate all'utilizzo di vaccini plasmaderivati. Sarà utile una ulteriore politica di sensibilizzazione per promuovere una diffusione ancora più capillare di questa efficace strategia preventiva, ormai indispensabile in ogni centro moderno di dialisi. 


\section{Bibliografia}

1. Kohler H, Arnold W, Renschin $\mathrm{G}$, et al. Active hepatitis $B$ vaccination of dialysis patients and medical staff. Kidney Int 1984; 25: 124-8.

2. Jacobs C, Brunner FP, Chantler $\mathrm{C}$, et al. Combined report on regular dialysis and transplantation in Europe. Proc Eur Dial Transpl Ass 1978; 14: 367.

3. Brynger H, Brunner FP, Chantler C, et al. Combined report on regular dialysis and transplantation in Europe. Proc Eur Dial Transpl Ass 1980; 16 : 2-86.

4. Wright $R$, Millword-Sadler GH, Bull FG. Acute Viral Hepatitis. In: Wright $R$, Millword-Sadler GH, Alberti $\mathrm{K}$ eds. Liver and Billary disease. London: Bailliere, Tindal publisher $1985 ; 677-768$.

5. Brown SE, Stanley C, Howard CR, Zuckerman, AJ, Steward MW. Antibody responses to recombinant and plasma derived hepatitis $\mathbf{B}$ vaccine. $\mathbf{B r}$ Med J 1986; 292: 159-62.

6. Papaevangelou G, Dandalos E, Roumeliotou-Karayannis A, Richardson SC. Immunogenity of recombinant Hepatitis B vaccine. The Lancet 1985: 455-6.

7. Crosnier J, Jungers $P$, Corouce $\mathrm{AM}$ et al. Randomimised placebo-controlled trial of hepatitis B surface antigene vaccine in French haemodialisis units. The Lancet 1981: 797-800.

8. Stevens CE, Szmuness W, Goodman AL, Weseley SA, Fotino M. Hepatitis B vaccine: immune responses in hemodialysis patients. Lancet 1980:
1211-3.

9. Weber DJ, Rutala WA, Samsa GP et al. Obesity as a predictor factor of poor antibody response to hepatitis B plasma vaccine. JAMA 1985; 254: 3187-9.

10. Ukena T, Esber H, Bassette $R$ et al. Site of infection and response to hepatitis $B$ vaccine. New Engl J Med 1985; 313: 579-80.

11. Steketee RW, Ziarnik ME, Davis JP. Seroresponse to hepatitis $B$ vaccine in patients and staff of renal dialysis centers, Wisconsin. Am J Epidemiol 1988; 127: 772-82.

12. Mark A, Kane MD, Stephen C, et al. Antibody to hepatitis B surface antigen and screening before hepatitis B vaccination. JAMA 1985; 103: 7913.

13. Rower P, Willems WR, Breidenbach $T$, et al. Seroconversion rate, hepatitis $B$ vaccination, hemodialysis and zinc supplementation. Kidney Int 1987; 32: 149-52.

14. Quiroga JA, Carreno V. Interferon and hepatitis $B$ vaccine in haemodialysis patients. Lancet 1989: 1264.

15. Zaruba K, Grob PS, Bolla K. Thymopentin as adjuvant theraphy to hepatitis $B$ vaccination in formerly non or hyporesponding hemodialysis patients. Surv Immunol Ris 1985; 4: 102-6.

16. Michel P, Janin G, Yafi S, et al. Improvement of immuno response in dialysis patients to Hevac B vaccine after multiple injections of vaccine. Proc EDTA 1985; 22: 1077-82.

17. Brugnora M, Radicio JL, Del Rio G, Esteban Mur R. Hepatitis: the disease and its prevention. Atti del simposio. Wa- shington 21 ottobre 1988.

18. Centers for disease controll. Update on hepatitis B prevention. MMVR 1987; 36: 354-8.

19. Guan R, Choong L, Tay H. Hepatitis $B$ vaccination in chronic renal failure patients undergoing renal dialysis using a recombinant hepatitis $B$ vaccine. J Gastroenterol Hepatol 1989; 4: 14.

20. Ponticelli C. Prevenzione dell'epatite B nei centri dialisi. Giornale Italiano di Nefrologia 1990; 7: 7-10.

21. Seaworh B, Drucker J, Starling $J$, et al. Hepatitis B vaccines in patients with chronic renal failure before dialysis. J Infect Dis 1989; 157: 332-337.

22. Street AC, Starling J, Dennis VW, Hamilton JD. Hepatitis B vaccination in pre dialysis renal failure patients. 3 year follow-up and results of booster vaccination. Clin Res 1989; 37 $326 \mathrm{~A}$.

23. Senneseal J, Van Der Niepen $P$, Verbeelen DL. Treatment with recombinant human erythropoietin increases antibody titers after Hepatitis B vaccination in dialysis patients. Kidney Int 1991; 40: 121-8. 